\title{
Endometriosis: A Clinicopathological Study in a Tertiary Care Hospital
}

\author{
Shubha P. Bhat ${ }^{1}$ Sajitha Kaliyat ${ }^{1}$ Kishan Prasad Hosapatna Laxminarayana ${ }^{1}$ \\ Teerthanath Srinivas ${ }^{1} \quad$ Krishna Prasad Holalkere Venugopala ${ }^{1} \quad$ Jayaprakash Kubalady Shetty ${ }^{1}$
}

1Department of Pathology, K.S Hegde Medical Academy, Nitte-Deemed to be University, Mangalore, Karnataka, India
Address for correspondence Shubha P. Bhat, MD, Department of Pathology, K S Hegde Medical Academy, Nitte- Deemed to be University, Mangalore, Karnataka, 575018, India (e-mail: bhatshubha_257@rediffmail.com).

\begin{abstract}
Keywords

- endometriosis

- site

- diagnosis

- treatment

Background Endometriosis (EM) is an estrogen-dependent disease characterized by the presence of endometrial gland and stroma outside the uterus. It is commonly seen in the reproductive age group and is a leading cause of infertility. Patients present with chronic pelvic pain, menstrual abnormalities, dyspareunia, or mass per abdomen. Surgical excision is the treatment of choice with postoperative medications and progesterone agents.

Aims and Objectives This study aims at determining the distribution of EM in terms of age, parity, symptoms, site, morphological features. We also analyzed the management of EM in these cases.

Methodology This is a retrospective study done in a tertiary care hospital in Mangalore, India. The case records of 77 histopathologically confirmed cases of EM during the 7 years between June 2012 and May 2019 were studied. Demographic profiles, parity, presenting complaints, site of the lesion, morphological features, treatment given, follow-up, and recurrence were noted and analyzed.

Results Of the 77 patients with EM, the most common age group was the fourth decade $(40 \%)$. The majority $(31,40.25 \%)$ of the patients in this study were nulliparous. Chronic pelvic pain was the most common symptom. The most frequent site was ovary. Conclusion The most frequent site of EM is ovary, leading to infertility. With the advent of laparoscopy, early diagnosis and excision have been feasible. The histopathological examination should be done for confirmation. Awareness of this disease among health care professionals is necessary whenever a woman in reproductive age presents with chronic pelvic pain and infertility.
\end{abstract}

\section{Introduction}

Endometriosis (EM) is the presence of endometrial tissue comprising glands and stroma located outside the uterus. ${ }^{1}$ Being one of the leading causes of infertility, 25 to $50 \%$ of infertile women have EM, and $30 \%$ to $50 \%$ of women with EM are infertile. ${ }^{2}$ The exact prevalence of EM is difficult to estimate, as many cases go underdiagnosed and the diagnosis requires invasive testing. Estimates ranges up to $10 \%$ among general population, 0.5 to $5 \%$ among fertile women, and 25 to $40 \%$ in infertile women. ${ }^{3}$ Retrograde menstruation, coelomic metaplasia, embryonic rests, lymphovenous dissemination, and transformation of endometrial stem cell theories are the pathophysiological mechanisms of
DOI https://doi.org/ 10.1055/s-0040-1720946 ISSN 0974-2727.
C 2020. The Indian Association of Laboratory Physicians.

This is an open access article published by Thieme under the terms of the Creative Commons Attribution-NonDerivative-NonCommercial-License, permitting copying and reproduction so long as the original work is given appropriate credit. Contents may not be used for commercial purposes, or adapted, remixed, transformed or built upon. (https://creativecommons.org/licenses/by-nc-nd/4.0/)

Thieme Medical and Scientific Publishers Pvt. Ltd., A-12, 2nd Floor, Sector 2, Noida-201301 UP, India 
EM. ${ }^{1,4}$ Although EM can be seen anytime between premenarche to postmenopause, it is foremost a disease seen in women of reproductive age. Common sites of EM include the cesarean section and episiotomy scars, ovaries, pouch of Douglas, uterosacral ligaments, vesicouterine pouch, urinary bladder, outer surface of the uterus, fallopian tubes, and round ligament. It is also seen in rectovaginal septum, rectosigmoid colon, appendix, cecum, distal ileum, omentum and, rarely, in pleura, lungs, diaphragm, and brain. ${ }^{5,6}$ The most common symptoms of EM are chronic pelvic pain, dysmenorrhea, dyspareunia, and infertility. ${ }^{7}$ Elevated peritoneal or serum CA 125 level measured during the menstrual phase is a useful marker of this disease. ${ }^{8}$ Laparoscopy, combined with the histopathological examination, is considered the gold standard for diagnosis. ${ }^{7}$ This retrospective study is aimed at determining the distribution of EM in terms of age, parity, symptoms, site, and morphological features. We also attempted to study the modes of management of EM.

\section{Materials and Methods}

This is a retrospective study done in a tertiary care hospital. Approval from the Institutional Ethics Committee was obtained. The case records of 77 histopathologically confirmed cases of EM during the 7 years between June 2012 and May 2019 were retrieved. Details about the demographic profile, parity, presenting complaints, site of the lesion, morphological features, and treatment received were recorded and analyzed. Follow-up details and recurrent cases were also noted.

\section{Results}

The study involved 77 females with EM. The majority of the patients were in the age group of 40 to 49 years (40\%), followed by 30 to 39 years (30\%), and 20 to 29 years (17\%). The youngest patient in our study was an 18 -year-old girl. The majority (40.25\%) of the patients in this study were nulliparous, followed by P2 L2 (23.37\%) and P1 L1 (19.48\%). We had two patients who were primigravida with tubal ectopic gestation and EM. There was one patient each (1.29\%) with parity of P4 L4 and P5 L5, respectively. There were six patients (7.79\%) with a history of abortions.

The presence of chronic pelvic pain was the most common complaint, which was seen in $50 \%$ of cases, followed by dysmenorrhea (30\%) and menorrhagia (14\%). Other complaints included mass per abdomen, nodule, wound gaping, infertility, irregular cycles, polymenorrhea, dysfunctional uterine bleeding (DUB), bleeding per vagina, dyspareunia, cough with expectoration, and breathlessness (-Table $\mathbf{1}$ ). The youngest patient in our study had left ovarian EM, complained of primary infertility, and was found to be having a rudimentary uterus on examination. - Table 2 shows the distribution of EM among the study subjects. Our study showed ovary as the most common site of EM (68\%), with the involvement of bilateral ovaries in $24 \%$ cases. Left and right ovaries were equally involved in $22 \%$ of cases. Fallopian tubes (31\%),
Table 1 Symptoms

\begin{tabular}{|l|l|l|}
\hline Symptoms & Number & Percentage \% \\
\hline Chronic pelvic pain & 38 & 50 \\
\hline Dysmenorrhea & 23 & 30 \\
\hline Dyspareunia & 2 & 3 \\
\hline Menorrhagia & 11 & 14 \\
\hline Infertility & 5 & 7 \\
\hline Nodule/wound gaping & 6 & 8 \\
\hline Mass per abdomen & 7 & 9 \\
\hline Polymenorrhea & 3 & 4 \\
\hline Irregular cycles & 4 & 5 \\
\hline DUB & 5 & 7 \\
\hline Cough and breathlessness & 1 & 1.5 \\
\hline
\end{tabular}

Abbreviation: DUB, dysfunctional uterine bleeding.

Table 2 Sites involved

\begin{tabular}{|l|l|l|}
\hline Site & Number & Percentage \% \\
\hline Left ovary & 17 & 22 \\
\hline Right ovary & 17 & 22 \\
\hline Bilateral ovaries & 18 & 24 \\
\hline Fallopian tubes & 24 & 31 \\
\hline Abdominal scar & 3 & 4 \\
\hline Appendix & 1 & 1.5 \\
\hline Pleura & 1 & 1.5 \\
\hline $\begin{array}{l}\text { Anterior and posterior surface of } \\
\text { the uterus }\end{array}$ & 1 & 1.5 \\
\hline Umbilicus/anterior abdominal wall & 5 & 7 \\
\hline Cervix & 2 & 3 \\
\hline Ureter & 2 & 3 \\
\hline
\end{tabular}

umbilicus/anterior abdominal wall (7\%), and scar EM (4\%) were the other sites. Rare sites of EM in our study included ureter (3\%), pleura, and appendix (1.5\% each).

Serum CA 125 assessments were done in 13 patients of EM, of which nine showed elevated levels and four showed normal levels.

Out of 77 patients, 42 (55\%) underwent total abdominal hysterectomy with bilateral salpingo-oophorectomy (TAH $\left.{ }^{+} \mathrm{BSO}\right)$. Out of these, 16 patients had adenomyosis. Excision of the endometriotic lesion by laparotomy was done in $22 \%$ with intra-abdominal/pelvic sites. Ovarian cystectomy was performed in $12 \%$ of cases. - Table $\mathbf{3}$ shows the various treatment modalities of EM in our study.

EM was an incidental finding in four cases: in the tubal stump of a patient with a prolapsed uterus, in the fallopian tube of a patient with carcinoma ovary, and fallopian tubes of two patients with ectopic pregnancy.

Associated findings included simple hyperplasia of endometrium in tubo-ovarian EM (four cases), sigmoid fistula, seromucinous cystadenoma, serous cystadenoma, and mucinous cystadenoma (one case each). 
Table 3 Surgery performed

\begin{tabular}{|l|l|l|}
\hline Type of surgery & Number & Percentage \% \\
\hline TAH + BSO & 42 & 55 \\
\hline Laparoscopic ovarian cystectomy & 9 & 12 \\
\hline $\begin{array}{l}\text { Tubectomy (for ruptured ectopic } \\
\text { gestation) }\end{array}$ & 2 & 3 \\
\hline Excision by laparotomy & 17 & 22 \\
\hline Local excision & 8 & 10 \\
\hline Appendectomy & 1 & 1.5 \\
\hline $\begin{array}{l}\text { Ureteric neocystostomy and } \\
\text { reimplantation }\end{array}$ & 2 & 3 \\
\hline VATS biopsy & 1 & 1.5 \\
\hline
\end{tabular}

Abbreviations: TAH +BSO, total abdominal hysterectomy with bilateral salpingo-oophorectomy; VATS, video-assisted thoracoscopic surgery.

\section{Discussion}

EM affects an estimated 89 million women of reproductive age worldwide. ${ }^{9}$ Discovered more than 100 years ago at the beginning of the 20th century, with the common name "adenomyosis externa," it is Thomas Cullen who deserves the credit. The term "endometriosis" was first used by John A. Sampson when observing bleeding of the lesions in the ovary while operating on women at the time of menstruation. ${ }^{4}$ Breus used the term "chocolate cyst" for the first time, while Runge provided a detailed description of endometrioma. Mayer described EM in an abdominal scar and intestinal EM. ${ }^{5}$ In 1860, Karl Von Rokitanski was the first to identify EM histologically under the microscope. 6,9

Various theories have been proposed in the etiopathogenesis for EM. Retrograde menstruation with regurgitation of endometrial cells into the peritoneal cavity still stands as the best explanation for most ectopic implants. The theory of coelomic metaplasia has also gained attention, which is based on the fact that the coelomic epithelium is the common precursor of endometrial and peritoneal cells. Embryonic rests theory hypothesizes that Mullerian remnants can differentiate into endometrial tissue. ${ }^{1}$ Also, to explain the cause of implants in distant sites, the theory of lymphatic/vascular microembolization and mechanical dissemination has been proposed. ${ }^{10}$

The average age at diagnosis of EM is 28 years, with risk increasing beyond 30 years and peaking at early to mid-40 years. There is a high concordance of the disease among identical twins. Familial predisposition with no clear Mendelian inheritance but rather multifactorial polygenic traits has been identified. Early menarche ( $<11$ years of age), short menstrual cycles ( $<27$ days), substantial and long-lasting bleeding, reduced parity, and reduced lifetime duration of lactation are known menstrual parameters. Bodyweight, body mass index, and waist to hip ratio have been inversely related to EM. Increased caffeine and alcohol consumption have been associated with increased risk, while regular exercise reduces the risk of EM. Exposure to polychlorinated biphenyl and dioxin has been associated with EM, while the use of oral contraceptive pills has slightly decreased prevalence. ${ }^{3,11}$
In our study, age ranged from 18 to 57 years. The majority of the patients were in the age group of 40 to 49 years (40\%), followed by 30 to 39 years (30\%), and 20 to 29 years (17\%). The youngest patient was an 18-year-old girl. The mean age of EM in studies done by Amaral et al, Satyanarayana et al, and Mishra et al was $29.2 \pm 5.6,29.4 \pm 7.7,29 \pm 4.3$ years, respectively. ${ }^{2.5,8}$ Most of our patients were symptomatic in their fifth decade of life, presenting with menstrual abnormalities $(n=23)$ and chronic pelvic pain $(n=15)$. Those with primary infertility seek medical advice much earlier than chronic pelvic pain. Hence, the most common age group of EM depends on the symptoms produced and the time of seeking healthcare assistance.

Adolescent EM can occur in association with obstructive Mullerian duct anomalies, with an estimated incidence being 11 to $40 \% .{ }^{12}$ The lesions are located in peritoneum and ovaries, with peritoneal implants categorized into early-active (red, glandular), advanced (black, puckered), and healed (white, fibrotic)..$^{13}$ Pathogenetic mechanisms of premenarcheal and adolescent EM are advancing. Stimulation of angiogenesis in endometrial implants is necessary for activation and survival, which is brought about by expression of vascular endothelial growth factor (VEGF) through endometrial cells, estrogenic effect of maternal steroids, and prepubertal estrogen. ${ }^{14}$ It is also said that the source of these implants is retrograde neonatal uterine bleeding, which occurs as degenerative, regressional changes in endometrium, leading to bleeding due to diapedesis, and these act as endometrial stem cells in the peritoneal cavity. ${ }^{15}$ We had three patients in the adolescent age group; all of them had EM of the ovary. Two of them came with primary infertility, of which one had a rudimentary uterus.

In adults, in addition to peritoneal EM and ovarian endometriomas, adenomyotic lesions or deep EM also occur. Deep EM is progressive EM with smooth muscle metaplasia, which is strongly associated with pelvic pain. ${ }^{16}$ Some of the pathogenetic mechanisms of adult EM include exaggerated menstrual preconditioning, endometrial dysfunction, and increased nerve fiber density in endomyometrium, causing pain and myometrial junctional zone hyperplasia. EM and adenomyosis have been linked in severe EM, which could explain the persistence of pain after extensive surgery for EM. ${ }^{4}$ In our study, 42 patients underwent $\mathrm{TAH}^{+} \mathrm{BSO}$, of which 16 patients had adenomyosis in addition to EM.

In pregnancy, complications include late miscarriage, preterm birth, and intrauterine growth restriction. ${ }^{17}$ We had two patients who were primigravida with tubal ectopic gestation and EM.

There is a high risk of premature menopause in patients with EM, which is associated with infertility. These women are treated with hormone replacement therapy (HRT). The use of HRT raises concerns like recurrence of pain, need for surgery, and $1 \%$ malignant transformation of residual EM. ${ }^{18}$ The most common location of EM in these patients is ovary, followed by urinary tract, skin, intestines, sigmoid, rectum, inferior vena cava, and vagina. The use of tamoxifen in these patients should be cautious, as it has an estrogenic effect on the endometrium in contrast to the antiestrogenic action on the breast. Postmenopausal EM occurring years after menopause in the absence of exogenous hormones is 
due to the conversion of adrenal androgen into estrogen after surgery or coelomic metaplasia in untreated patients. ${ }^{4}$ We had one patient on HRT, presenting with postmenopausal bleeding due to simple hyperplasia of endometrium without atypia. The left ovary showed serous cystadenoma with EM.

Symptoms depend on the location of implants. The most common symptoms of EM are dysmenorrhea, dyspareunia, chronic pelvic pain, and infertility. Approximately one-third of patients with EM are asymptomatic. EM of the appendix can present with pelvic pain, Malena, lower gastrointestinal bleeding, or intussusception. ${ }^{6}$ In thoracic endometriosis syndrome (TES), chest pain, dyspnea, hemoptysis, and cough are the presenting complaints. ${ }^{9}$ The cause of infertility in EM is attributed to the distortion of pelvic anatomy by pelvic and peritubal adhesions, endocrine and ovulatory abnormalities, altered peritoneal function with increased peritoneal fluid volume, and altered hormonal and cell-mediated functions in the endometrium which affect embryo implantation. ${ }^{5}$ The presence of chronic pelvic pain was the most common complaint noted in our study, which accounted for $50 \%$ of cases, followed by dysmenorrhea (30\%) and menorrhagia (14\%). Primary infertility was the presenting complaint in only five cases (7\%). However, when we assessed the parity, 31 patients (40.25\%) were nulliparous. In a study by Amaral et al., dysmenorrhea was the most common symptom (91\%), followed by infertility (71.4\%) and dyspareunia (57\%). ${ }^{8}$ Almeida Filho et al. ${ }^{7}$ also found dysmenorrhea as a common symptom in their study. Mishra et al., ${ }^{8}$ in their research, found primary infertility as the common complaint in $75 \%$ of cases and secondary infertility in $25 \%$. These studies had a mean age group of patients much younger in the third decade. Our research found most patients in their 5th decade of life. Patients with primary infertility and dysmenorrhea present much earlier compared with chronic pelvic pain.

Positive clinical findings like tenderness on examination, fixed retroverted uterus, or pelvic mass should always raise the index of suspicion toward EM. ${ }^{2}$ Elevated peritoneal or serum CA 125 level measured during the menstrual phase is a useful marker of this disease. The levels are found higher in peritoneal fluid in superficial disease, whereas in deep lesions, it is high in serum. Levels of CA 125 in the peritoneal fluid is considered a more sensitive indicator of disease and in the detection of early-stage EM. ${ }^{8}$ However, CA 125 levels are elevated in certain gynecological conditions like cancers of ovary and cervix, pelvic inflammatory disease, and ovarian hyperstimulation syndrome. Nongynecological conditions where CA 125 is raised are liver cirrhosis, pulmonary tuberculosis, interstitial lung disease, congestive heart failure, atrial fibrillation, and pericardial disease. ${ }^{19}$ Two-dimensional or three-dimensional transvaginal ultrasound, transvaginal endoscopy, and transvaginal hydrolaparoscopy are helpful in early diagnosis and ablating endometriomas with a maximum diameter of $3 \mathrm{~cm} .{ }^{5}$ Laparoscopy, combined with the histopathological examination, is considered the gold standard for diagnosis. ${ }^{7}$

Endometriotic lesions are classified into subtle, typical, cystic, and deep. Macroscopically visible, nonpigmented lesions without surrounding sclerosis were called "subtle." They were considered the early lesions after implantation and are expected to be found intermittently in all women if screened by laparoscopy and hence should not be considered a disease. ${ }^{11}$ The histological diagnosis of EM is readily made when both endometrial glands and/or stroma are present outside the uterus. Diagnosis is sometimes difficult in long-standing cases in which tissue is obscured by secondary fibrosis, or there is the only hemorrhage with hemosiderin-laden macrophages. ${ }^{6}$ Differential diagnoses of ovarian EM include a corpus luteal cyst with secondary hemorrhage. ${ }^{11}$ In scar EM, one must exclude abscess, suture granuloma, hematoma, desmoids tumor, nodular fasciitis, and primary and metastatic cancer. Fine needle aspiration cytology (FNAC) helps in confirming the preoperative diagnosis of scar EM. Cytological features of scar EM vary with cyclical hormonal changes and show epithelial cells with small round-to-oval nuclei and bland chromatin in sheets along with spindle-shaped stromal cells in the proliferative phase, with a gradual increase in cell size with cytoplasmic vacuolations in the secretory phase. Background usually contains inflammatory cells and hemosiderin-laden macrophages. ${ }^{5}$ Out of eight cases of abdominal wall/scar EM in our study, FNAC was performed and confirmed the diagnosis in three cases.

Endometriosis typically appears as superficial powder burn or gunshot lesions on the ovaries, serosal surfaces, and peritoneal black, dark brown, or bluish puckered lesions, nodules, or cysts containing old hemorrhages surrounded by fibrosis. ${ }^{1}$ Ovarian cystic EM contains thick tar-like chocolate fluid and is usually confused with cystic corpora lutea. They are adherent to the ovarian fossa, tubes, and bowel and contain high CA 125 in the chocolate fluid. ${ }^{11}$ Our study showed ovary as the most common site of EM (68\%), with the involvement of bilateral ovaries in $24 \%$ cases ( - Fig. 1). Histology showed endometrial glands and stroma surrounded by hemorrhage, hemosiderin, and fibrosis ( - Fig. 2). Ovaries with peritoneal involvement were the most common site of EM $(49.7 \%)$ in a study done by Gruppo Italiano. ${ }^{20}$

Fallopian tubes were the next common site of EM (31\%). Endometrial glands and stroma were seen in the wall of the

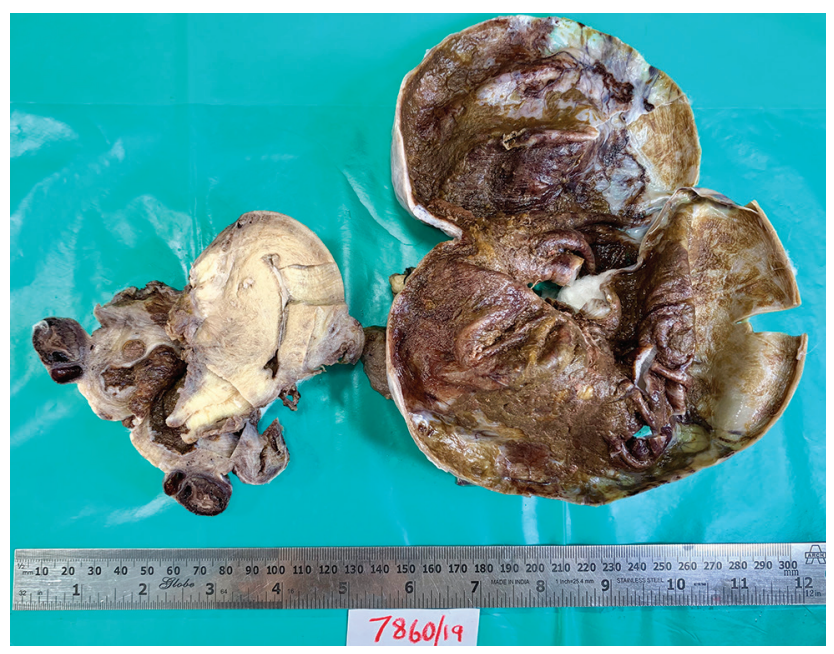

Fig. 1 Gross specimen of hysterectomy with bilateral salpingooophorectomy showing bilateral chocolate cysts in the ovary. 
fallopian tube (-Fig. 3). EM of fallopian tube was an incidental finding in a bilateral tubal stump in a patient with a prolapsed uterus, in a patient with carcinoma ovary, and two patients with ectopic pregnancy (-Fig. 4).

EM occurring in the postoperative scar is rare, with the first case reported by Meyer in 1903. It is thought that EM of umbilicus occurs due to iatrogenic translocation of intraperitoneal endometrial tissue during abdominal surgeries. However, cases without prior history of abdominal surgeries have been noted. ${ }^{1}$ Our study had three cases (4\%) of scar endometriosis, following Caesarean section, who came with

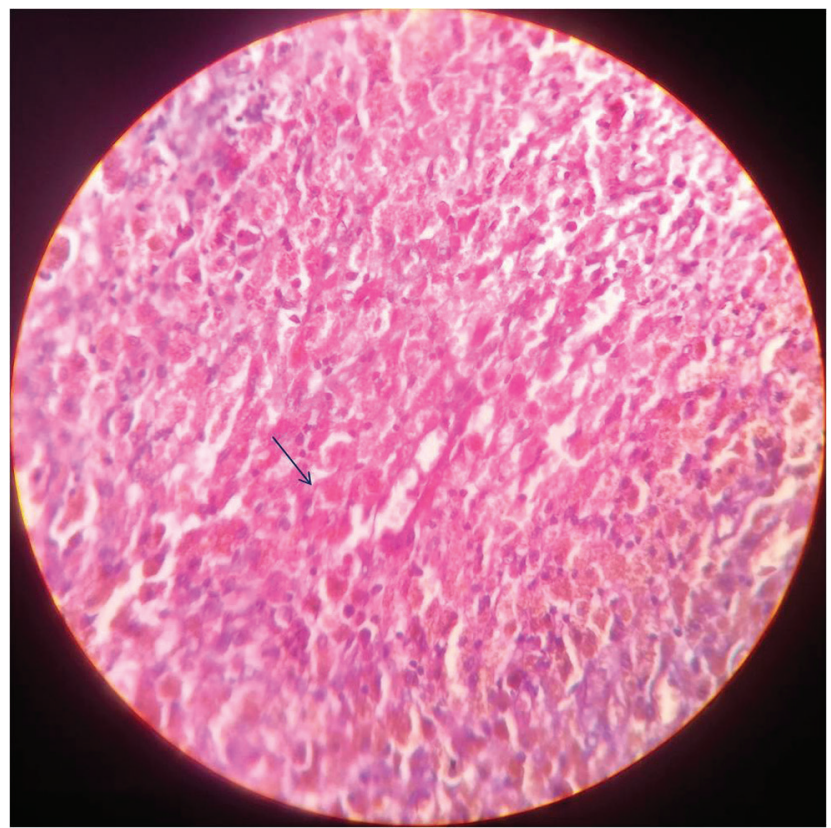

Fig. 2 Microscopy of chocolate cyst ovary showed endometrial glands and stroma surrounded by hemosiderin-laden macrophages (arrow), hemotoxylin and eosin (H\&E), 10×.

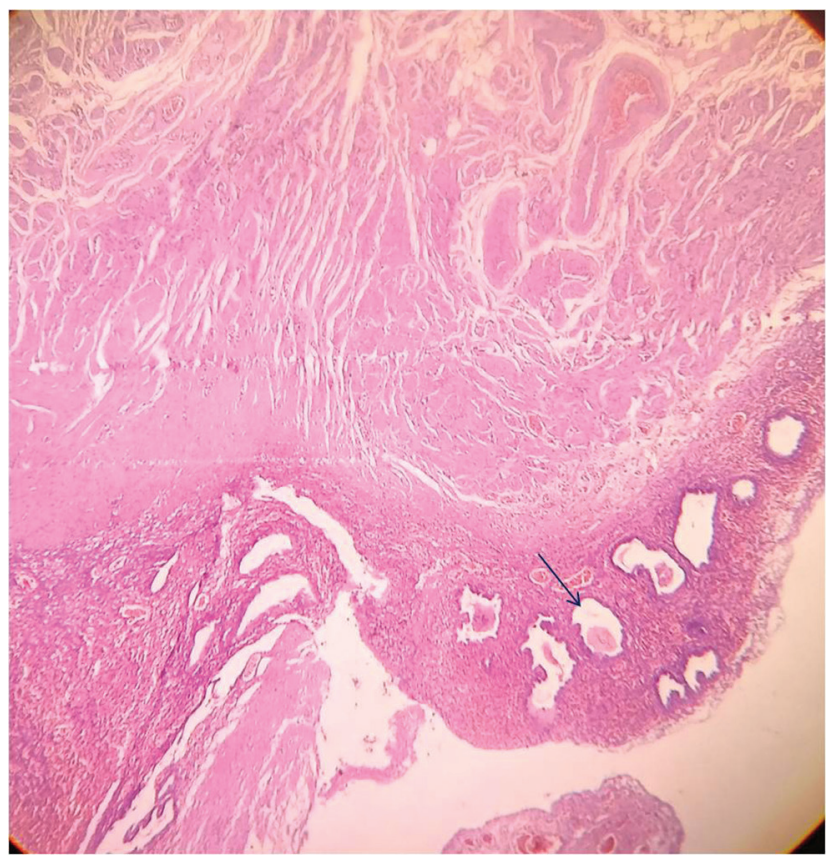

Fig. 3 Endometrial glands and stroma in tubal serosa (arrow), hemotoxylin and eosin (H\&E), 10×. wound gaping and bleeding, and five cases (7\%) of EM at the umbilicus and anterior abdominal wall.

Urinary tract involvement in EM is between 1 to $5 \%$, with the bladder being the most common site, followed by ureter, kidney, and urethra with a ratio of $40: 5: 1 .^{1,5}$ The present study had two cases (3\%) of ureteral EM (-Fig. 5). One patient had associated bilateral ovarian EM.

The involvement of the gastrointestinal tract is estimated to be between 3 to $37 \%$, with the rectosigmoid being the most common site (72\%), followed by the rectovaginal septum (13\%), small intestine (7\%), cecum (3.6\%), and appendix (3\%). It is a common incidental finding during laparoscopy or laparotomy.

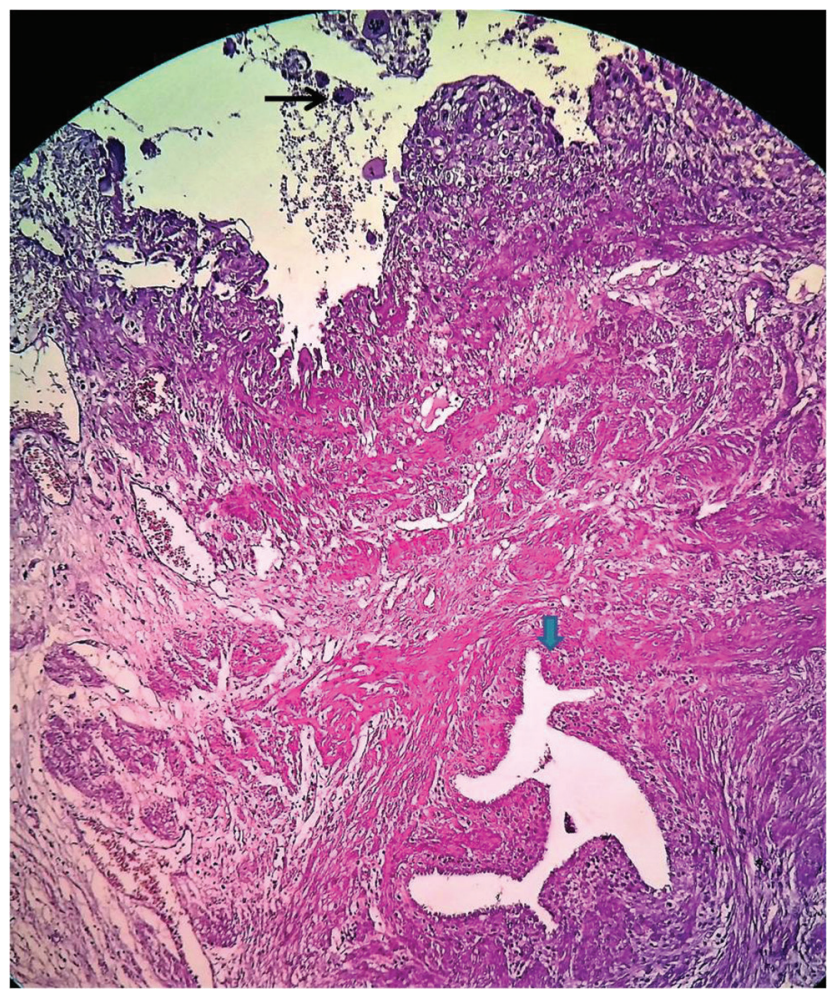

Fig. 4 Ectopic tubal gestation showing syncitiotrophoblastic giant cells in lumen (arrow) with endometriosis in the wall (arrowhead), hemotoxylin and eosin (H\&E) staining, 10x.

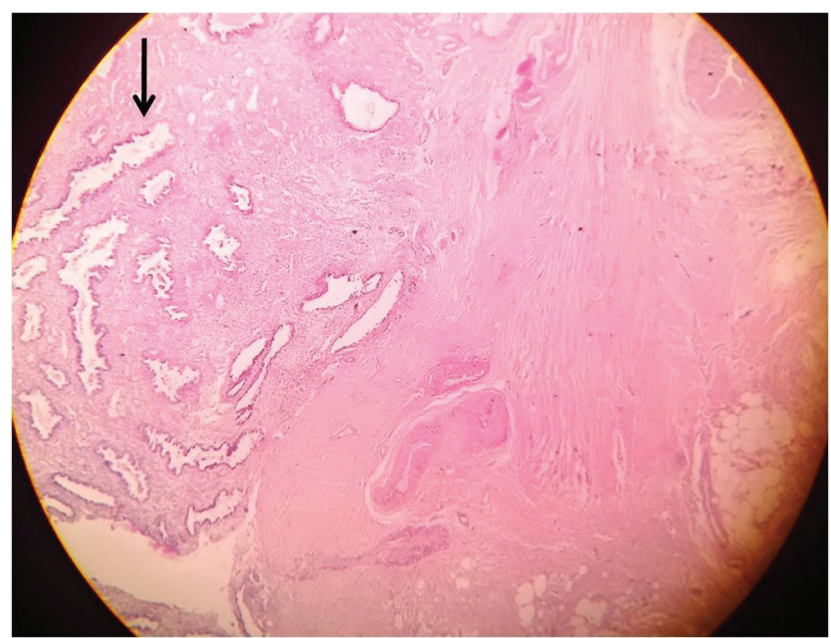

Fig. 5 Endometriosis of ureteric wall (arrow), hemotoxylin and eosin (H\&E) staining, $10 \times$. 
Endometriosis must be suspected when an inverted or bulbous appendiceal orifice is noted at colonoscopy. Appendectomy is the treatment of choice. Superficial spots of EM can be vaporized with carbon dioxide, while extensive lesions need small bowel resection and anastomosis. EM usually involves the serosa and subserosa, with more proximal involvement up to mucosa in severe cases. ${ }^{6}$ Our study showed evidence $(1.5 \%)$ of appendiceal EM in a patient who complained of pain at the right lower abdomen. Histology showed endometrial glands and stroma in the muscularis propria (-Fig. 6).

EM occurring in the lung parenchyma, pleura, and other parts of the airway is called TES, which clinically presents as catamenial pneumothorax, catamenial hemothorax, catamenial hemoptysis and lung nodules. Of the total women with TES, 50 to $85 \%$ have concomitant pelvic EM..$^{21}$ Pathogenesis of TES includes diaphragmatic fenestrations which could be congenital or due to direct erosion by endometrial implants through which they travel to thorax from peritoneal cavity, coelomic metaplasia of pleural epithelium, and lymphatic/ vascular microembolization. Video-assisted thoracoscopic surgery (VATS) allows direct visualization of deposits on lung and diaphragmatic surfaces. It also permits resection of implants, closure of diaphragmatic fenestrations, and abrasive mechanical or chemical pleurodesis. ${ }^{10}$ We had a 30-year-old female patient who was referred to us for repeated right-sided pleural effusion with lung collapse. The patient was infertile and had been on treatment for it. Ultrasound abdomen and pelvis showed normal ovaries with fluid in the pouch of Douglas and peritoneal cavity. Pleural

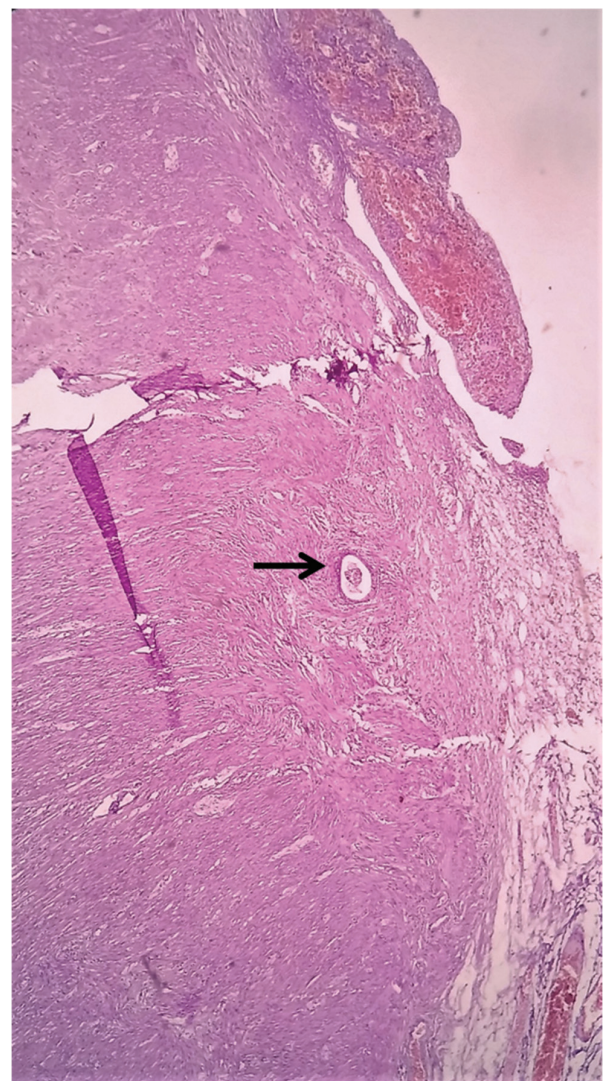

Fig. 6 Endometriosis in the wall of the appendix (arrow), hemotoxylin and eosin (H\&E) staining, 10x. tapping done in another hospital showed lymphocytic effusion with plenty of hemosiderin-laden macrophages; empirically, she was treated with antitubercular therapy. Biopsy of irregular nodules in costal parietal pleura seen through VATS ( - Fig. 7) showed endometrial glands and stroma with hemosiderin-laden macrophages under the mesothelial lining ( - Fig. 8a, b). Chemical pleurodesis with bleomycin was done and oral progesterone was advised.

Complications of EM are bleeding, causing adhesions and decreased fertility, increased risk of miscarriage and premature delivery, rupture of the endometriotic ovarian cyst, and obstruction in the intestine. ${ }^{1}$ Less than $1 \%$ of EM can undergo malignant transformation into endometrioid or clear cell carcinoma, with the ovary being the most common site. ${ }^{5}$ We did not detect any cases of EM with malignant transformation in our study.

Treatment of EM can be medical or surgical, depending on the severity of symptoms and patients' desire to restore fertility. Medical treatment involves hormonal manipulation of the menstrual cycle to create an amenorrheic state,

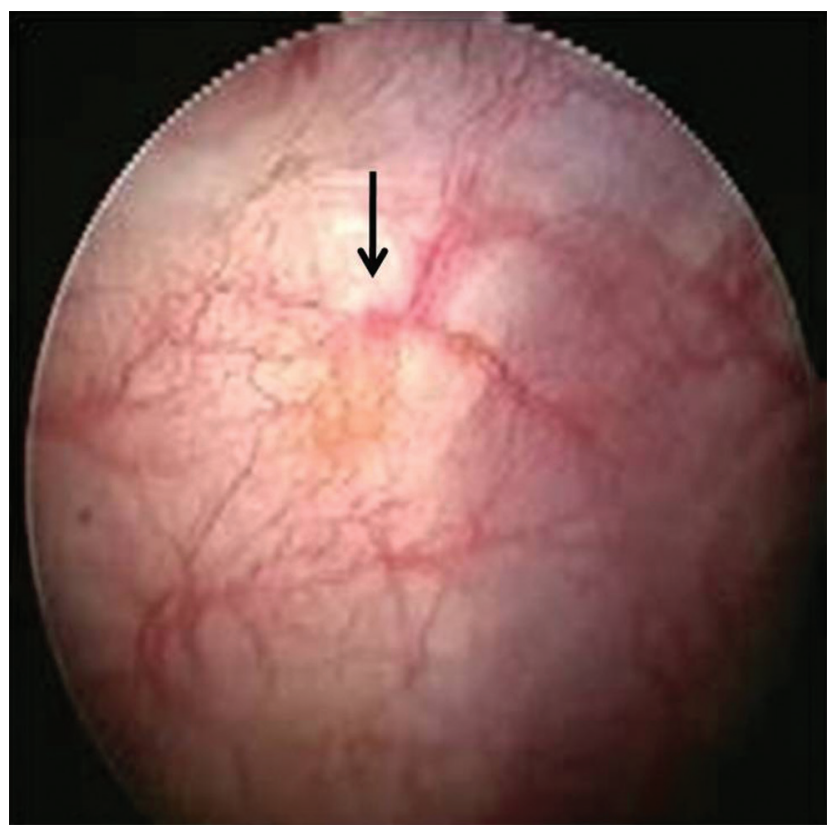

Fig. 7 Pleural endometriosis; video-assisted thoracoscopy showing reddish nodules on costal parietal pleura (arrow).
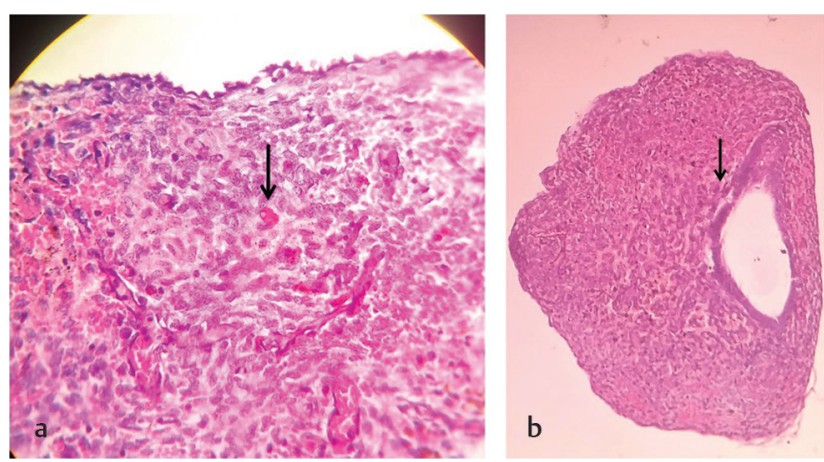

Fig. 8 (a) Endometriosis of pleura showing endometrial stromal cells and hemosiderin-laden macrophages (arrow) with an overlying mesothelial lining, hemotoxylin and eosin (H\&E) staining, 10×. (b) Endometrial gland in the pleura (arrow), hemotoxylin and eosin (H\&E) staining, 10×. 
thus producing an environment unfavorable to endometrial tissue. Danazol, progestational agents, gestrinone, oral contraceptives, and gonadotropin-releasing hormone ( $\mathrm{GnRH})$ agonists are conventionally used medical agents. ${ }^{3}$ Danazol prevents midcycle luteinizing hormone surge, causing anovulation which, in turn, decreases the secretion of estradiol. GnRH analogs downregulate the GnRH receptor, causing reversible hypogonadotropic hypogonadism. ${ }^{10}$ However, these medications are found to delay fertility instead of increasing pregnancy rates. Preoperative nasal application of GnRH agonists decreases inflammation and vascularization of EM and improves the surgical field. EM lesion ablation with adhesiolysis improves fertility in minimal-to-mild EM compared with diagnostic laparoscopy alone. ${ }^{3} \mathrm{TAH}+\mathrm{BSO}$ is done in patients who have completed their family and in cases of intractable pain. However, it does not address dormant endometrial implants that may become active with the administration of exogenous estrogen. ${ }^{10}$ Postoperative medical therapy achieves resorption of residual deposits that cannot be surgically removed, destruction of microscopic implants, and reduction of disease dissemination in case of endometrioma rupture. Assisted reproductive techniques (ART) are suggested for EM associated infertility. Intrauterine insemination with controlled ovarian hyperstimulation, limited to a maximum of three to four cycles, is beneficial but poses the risk of progression of ovarian EM. In vitro fertilization (IVF) technique is suitable for cases having impaired tubal function, male factor infertility, and/ or failure of other treatments. It is better to consider GnRH agonist treatment before IVF in cases of moderate-to-severe EM to enhance the pregnancy rate. ${ }^{3}$

Recurrence rates were different for varied types of EM lesions. It is around $20 \%$ for typical injuries, approximately $7 \%$ after stripping, 20\% after superficial destruction of cystic ovarian EM, and less than 5\% after excision of deep EM.

This study has extensively evaluated the clinical characteristics and diagnostic modality of EM. However, it is a retrospective study, and hence we wish more prospective studies to come up in the future, keeping the findings of our research in mind, which will significantly contribute to enhancing the diagnostic accuracy and prognosis of patients with EM.

\section{Conclusion}

EM is a chronic and recurrent disease predominantly found in women of childbearing age. The most frequent site of occurrence is ovary, leading to infertility and chronic pelvic pain. With the advent of laparoscopy, early diagnosis and excision have been feasible. This must be followed by medications with progesterone agents for the resorption of residual deposits. The histopathological examination should be done for confirmation. Awareness of this disease among healthcare professionals is necessary whenever a woman in reproductive age presents with chronic pelvic pain and infertility.

\section{References}

1 Agarwal N, Subramanian A. Endometriosis - morphology, clinical presentations and molecular pathology. J Lab Physicians 2010;2(1):1-9

2 Mishra VV, Gaddagi RA, Aggarwal R, Choudhary S, Sharma U, Patel U. Prevalence, characteristics and management of endometriosis amongst infertile women: a one year retrospective study. J Clin Diagn Res 2015;9(6):QC01-QC03

3 Ozkan S, Murk W, Arici A. Endometriosis and infertility: epidemiology and evidence-based treatments. Ann N Y Acad Sci 2008;1127:92-100

4 Brosens I, Puttemans P, Benagiano G. Endometriosis: a life cycle approach? Am J Obstet Gynecol 2013;209(4):307-316

5 Satyanarayana MA, Sushma V, Kumar KP, Kumar VS, Murthy KR, Asha T. Endometriosis: A clinicopathological study of 29 cases. J Clin Diagn Res 2018;12(5):5-9

6 Saleem A, Navarro P, Munson JL, Hall J. Endometriosis of the appendix: Report of three cases. Int J Surg Case Rep 2011; 2(2):16-19

7 Almeida Filho DP, Oliveira LJ, Amaral VF. Accuracy of laparoscopy for assessing patients with endometriosis. Sao Paulo Med J 2008;126(6):305-308

8 Amaral VF, Ferriani RA, Sá MF, et al. Positive correlation between serum and peritoneal fluid CA-125 levels in women with pelvic endometriosis. Sao Paulo Med J 2006;124(4):223-227

9 Rawala MS, Khaliq MF, Iqbal MA, Naqvi ST, Farhan K, Myers A, et al. A rare case of cyclical hemothorax: thoracic endometriosis syndromeCase Rep Pulmonol 2018;(e-pub ahead of print). doi: https://doi.org/10.1155/2018/9830797

10 Azizad-Pinto P, Clarke D. Thoracic endometriosis syndrome: case report and review of the literature. Perm J 2014;18(3): 61-65

11 Koninckx PR, Ussia A, Keckstein J, Wattiez A, Adamyan L. Epidemiology of subtle, typical, cystic, and deep endometriosis: a systematic review. Gynecol Surg 2016;13:457-467

12 Dovey S, Sanfilippo J. Endometriosis and the adolescent. Clin Obstet Gynecol 2010;53(2):420-428

13 Vasquez G, Cornillie F, Brosens IA. Peritoneal endometriosis: scanning electron microscopy and histology of minimal pelvic endometriotic lesions. Fertil Steril 1984;42(5):696-703

14 Oliveira FR, Dela Cruz C, Del Puerto HL, Vilamil QTMF, Reis FM, Camargos AF. Stem cells: are they the answer to the puzzling etiology of endometriosis? Histol Histopathol 2012; 27(1):23-29

15 Lin J, Xiang D, Zhang J-L, Allickson J, Xiang C. Plasticity of human menstrual blood stem cells derived from the endometrium. J Zhejiang Univ Sci B 2011;12(5):372-380

16 Cornillie FJ, Oosterlynck D, Lauweryns JM, Koninckx PR. Deeply infiltrating pelvic endometriosis: histology and clinical significance. Fertil Steril 1990;53(6):978-983

17 Stephansson O, Kieler H, Granath F, Falconer H. Endometriosis, assisted reproduction technology, and risk of adverse pregnancy outcome. Hum Reprod 2009;24(9):2341-2347

18 Soliman NF, Hillard TC. Hormone replacement therapy in women with past history of endometriosis. Climacteric 2006; 9(5):325-335

19 Tahmasebi F, Nath R, Sokolovsky N, et al. Incidental finding of raised CA125: a cause for concern? Crit Care Obst Gyne 2018;5(1):1-5

20 Gruppo Italiano per lo Studio dell'Endometriosi. Relationship between stage, site and morphological characteristics of pelvic endometriosis and pain. Hum Reprod 2001;16(12):2668-2671

21 Nair SS, Nayar J. Thoracic endometriosis syndrome: a veritable Pandora's Box. J Clin Diagn Res 2016;10(4):QR04-QR08

Conflict of Interest

None declared. 